\title{
Paleolítico Medio de aspecto postachelense en la depresión inferior del Guadalquivir
}

\author{
ENRIQUE VALLESPI *
}

Las investigaciones en curso de realización de las terrazas del Bajo Guadalquivir, Guadalete y Tinto y Odiel, de la depresión de La Janda y de la orla litoral atlántica implicada, permiten plantear las perspectivas de estudio de una secuencia regional del Paleolítico Inferior y Medio en la Baja Andalucía, con atribuciones dudosas a una etapa preachelense local y la existencia de una seriación achelense con probable Achelense Inferior o Medio Antiguo, Achelense Medio evolucionado, Achelense Superior y Final y un Paleolítico Medio todavía sin definir y sobre suya valoración inicial pretende reflexionar en esta nota, con la que me uno cordialmente al merecido homenaje al Profesor Eduardo Ripoll, con la evocación de una larga y permanente amistad.

Esta secuencia regional está vertebrada por las muestras de industrias extraídas del interior de los depósitos detríticos de las terrazas del Guadalquivir en la provincia de Sevilla, en cuyo sector Sevilla-Carmona se distinguen 12 niveles: T1 a T4 atribuidos al Pleistoceno Inferior y su transición, T5 a T8 al Pleistoceno medio y transición y T9 y T10 al Superior, correspondiendo T11 y T12 al Holoceno e Histórico, según nuestras investigaciones en curso (Díaz del Olmo y Vallespi, 1988). En su estimación actual, la seriación arqueológica de esta secuencia se inicia en los niveles T2 y T3, con industrias de un Paleolítico Inferior antiguo todavía por determinar, presenta un rico conjunto del Achelense Medio

\footnotetext{
* Universidad de Sevilla.
} 
evolucionado en un paleocanal (T4P) sobreimpuesto a T5, un potente Achelense Superior en T6 y T7, Achelense Final transicional en T8 y Paleolitico Medio, al que luego nos referiremos, en T9 y T10. Esta secuencia se extiende al Sector la Campana y afecta a las interrelaciones de los tramos inferiores del Corbones y el Genil, que están siendo estudiados por J. J. Fernández Caro y C. Pereda, respectivamente.

En el Guadalete hay industrias en conexión con una terraza alta del tramo inferior del río, en los alrededores de Jerez (Giles y Santiago, 1988), que, a mi modo de ver, deben atribuirse al Achelense Superior, y se han extraído asimismo muestras e industrias del interior de la terraza baja en el tramo medio-alto, pertenecientes al Paleolítico Medio (Vallespí, Álvarez, Díaz del Olmo, 1983-84), que también pasaremos después a considerar. De la depresión lagunar de La Janda tengo noticias del control reciente de evidencias de conexiones, debidas a J. R. Ramírez, V. Mateos y C. Fernández-Llebrez, actualmente en estudio.

De los rios Tinto y Odiel disponemos de varias localizaciones con muestras de industrias procedentes del interior de las terrazas (Castineira, García Rincón y otros, 1988; Rodríguez Vidal y otros, 1988). Del nivel más alto del Tinto procede un lote de piezas líticas que deben corresponder al Achelense Superior y de la terraza media de dicho río se ha extraído un lote de piezas de adscripción al Paleolítico Medio. Del Odiel hay asimismo una localización cuya posición estratigráfica y el aspecto de los materiales extraídos reclaman también la misma atribución al Paleolítico Medio y en una terraza del mismo río, en plena área litoral, un sondeo estratigráfico reciente ha librado una pequeña muestra de piezas atribuidas también con alguna duda a la misma época. Aparte queda alguna otra evidencia menos significativa de momento.

En depósitos continentales del litoral, el yacimiento de El Aculadero (Querol y Santonja, 1983) puede situarse en el Pleistoceno Medio avanzado, paralelas de este modo sus industrias al pleno Achelense, según la opinión actual de uno de sus excavadores (Santonja, e. p.). En el mismo litoral atlántico, algún otro indicio de conexión estratigráfica de industrias parece ampliar estas evidencias.

Resumiendo para nuestro propósito, vemos que en esta secuencia regional standard las industrias del Paleolítico Medio están representadas por los materiales extraídos del interior de los depósitos de las localizaciones con conexiones en las terrazas bajas del Guadalquivir, relativamente numerosas, en una localización en el Guadalete y en sendas localizaciones en el Tinto y Odiel, aparte de otra dudosa en este último 
río y de alguna evidencia sin aclarar, limitándonos exclusivamente a los materiales o referencias publicadas o de mi responsabilidad personal.

En el Guadalquivir, en el tramo Sevilla-Carmona, los niveles que nos afectan T9 y T10, presentan conexiones de industrias en cuatro y tres localizaciones respectivamente. En T9, en Morilla, Camino 24/25 y Toruño, con sólo 2 núcleos, 5 lascas, 1 canto tallado de filo bifacial y 2 diversos, y en Brenes un conjunto integrado por 11 núcleos, 47 lascas, 3 cantos tallados de filo unifacial, 1 punta levallois, 4 raederas, 1 perforador, 1 cuchillo, 1 muesca, 1 denticulado y 12 diversos. Las tres localizaciones con conexiones en T10, km. 111 de Carmona a Lora, Guadajoz y San José de la Rinconada, ha proporcionado 8 núcleos, 33 lascas, 9 cantos tallados de filo unifacial, 1 canto tallado de filo bifacial y 3 cantos tallados diversos. En la margen derecha del Guadalquivir, en nivel equivalente de T10 de la izquierda, hay cuatro localizaciones con conexiones arqueológicas, en Peones Camineros, Santa Iglesia, San José y Arroyo Gabino, que han librado 16 núcleos, 35 lascas y 1 canto tallado de filo unifacial.

Del interior de la terraza baja del Guadalete, en su tramo medio-alto, sector de Villamartín, proceden 1 núcleo, 3 lascas, 2 cantos de filo unifacial, 1 canto de filo bifacial, 2 cuchillos de dorso natural y 2 diversos (cantos tallados o núcleos). En el Tinto, del interior de una gravera del Apeadero de Niebla se han extraido 3 núcleos (dos de ellos discoides), 2 lascas, 2 cantos de filo unifacial, 1 canto de filo bifacial y 2 muescas retocadas, y del Odiel, de la localización de El Gritillo, asimismo de la terraza baja, 1 núcleo, 2 lascas, 2 cantos de filo unifacial, 1 muesca y 2 diversos nucleiformes.

Comprobada asi la existencia de Paleolítico Medio en dichas terrazas bajas fluviales y aunque de momento las muestras de industrias extraidas son totalmente insuficientes para cualquier cuantificación, podemos ensayar un acercamiento valorativo al hecho registrado.

En primer lugar queda clara la continuidad de la ocupación de dichas formaciones y del aprovechamiento consiguiente de sus cantos rodados como recurso exclusivo de talla: el predominio de los restos de taller, en proporción aceptable de núcleos y lascas, sobre las piezas transformadas en tipos, en casi todas las localizaciones, lo evidencia con claridad, aunque de momento no puedan definirse los propios lugares de los talleres, ya que se trata de niveles de ocupación, sin precisión posible por ahora. En este sentido se observa que las materias primas empleadas corresponden a la composición de los cantos rodados de las propias formaciones, comprobándose, en efecto, en el caso del Guadalquivir, un 
aprovechamiento selectivo de los cantos de sílex, a los que corresponde aproximadamente la mitad de las piezas registradas. En el Guadalete predomina la talla de los cantos de caliza, junto al uso mucho menos de los de lidita, arenisca y sílex, lo que también está en relación con los cantos rodados del depósito detrítico, y del mismo modo, tampoco hay uso preferente del silex en el Tinto y Odiel. El silex parece, por lo tanto, que se busca especialmente en las formaciones donde existe en mayor proporción.

Vistas en su conjunto, las industrias presentan una mixtificación generalizada de las técnicas de talla, con abundancia de núcleos de extracciones únicas o escasas y núcleos globulosos y de extracción regularizadas, siendo en éstos habituales los discoides y levallois; las series de lascas están constituidas consiguientemente por lascas de estilo clactoniense, ordinarias y levallois, y puntas pseudo y levallois. En el cuadro tipológico registrado predominan absolutamente los cantos tallados, sobre todo los de filo unifacial y en menor grado los de filo bifacial, de presencia generalizada en casi todas las localizaciones y que valorados en su conjunto de las muestras recogidas sobrepasan holgadamente la tercera parte del total de las piezas tipológicas, aunque tal valoración porcentual sea de momento circunstancial. De la lista normativa de tipos sobre lasca aparecen representados, además del de lasca y puntas pseudo y levallois, como hemos dicho, los tipos de raedera (simple, doble, convergente y transversal), perforador, cuchillo de dorso cortical, muesca y denticulado, aparte de los diversos, que engloban provisionalmente muchas piezas para determinar.

Todo ello, referido exclusivamente a los materiales procedentes del interior de los depósitos detríticos, queda de momento sin posible valoración porcentual, debido a la exigüidad de las muestras extraidas, cuyo balance tipológico se verá sin duda matizado con el estudio analítico pendiente y quedará en todo caso muy reforzado por las nutridas y más variadas series de superficies de las terrazas bajas en cuestión, entre cuyos materiales hay, por ejemplo, algún bifaz subtriangular y cordiformes más frecuentes.

Como interpretación cultural del Paleolítico Medio prefigurado inicialmente de este modo, en las terrazas fluviales de la depresión inferior del Guadalquivir, lo que de momento me interesa destacar es su aspecto de perduración achelense, claramente evidenciado y que, a mi modo de ver, constituye el primer rasgo definidor de las muestras de sus industrias controladas.

En efecto, si contemplamos la secuencia fluvial del Bajo Guadalquivir, donde, como venimos diciendo, se vertebra el proceso ahora cono- 
cido del Paleolítico Inferior y Medio de la Baja Andalucía, vemos que el desarrollo secuencial de sus industrias se comporta como un continuum tecnológico sobre cantos rodados, con tipos nucleiformes y de lascas, definido en síntesis por: la continuidad de los cantos tallados (choppers, choppings y cantos tallados diversos) en toda la secuencia (de T2 a T10, ambas terrazas inclusive), la presencia del bloque macrolitico de bifaces, hendedores y triedros en las terrazas medias-altas y medias (T4P a T8, inclusive), y, como rasgo incipientemente evidenciado, la matización creciente de los Grupos tipológicos de la lista normativa, desde las terrazas medias a las bajas (T6 a T10, inclusive).

En la interpretación de este proceso, que ha quedado expuesta al principio de estas notas, al Achelense Superior de T6 y T7 sucede un nivel, T8, Achelense Final, con Elephas antiquus y Equus hydruntinus y una industria con cantos tallados y representaciones de los tipos de bifaz, triedro, de hendedor (en superficie), raedera, cuchillo de dorso, muesca y diversos, en cuyo conjunto la talla de cuarcitas, prácticamente exclusiva en toda la secuencia anterior, cede al silex un 25 por 100 aproximadamente, constituyendo un horizonte transicional al de los dos niveles subsiguientes, T9 y T10, del Paleolítico Medio que comentamos, cuyo conjunto industrial está tallado al 50 por 100 aproximadamente en cuarcitas y en silex, lo que refuerza la continuidad que reclama la relación evidente de las respectivas industrias de ambos horizontes culturales. Este Paleolítico Medio aparece, en fin, como un complejo terminal de las industrias de la secuencia, matizado en dichos niveles T9 y T10 por un impacto musteriense, representado por la talla levallois de puntas y por determinados modos de retoque y tipos de raderas, principalmente.

Son precisamente a estas evidencias lo que vengo denominando Paleolítico Medio, o Musteriense, de graveras, y llamé en trabajos anteriores, sobre industrias de la submeseta Sur, Musteriense de tradición achelense genérico y no de facies, en contraposición con las facies del Musteriense clásico. Tal vez pueda concebirse como un simple musteroide, pero intuyo que se trata de algo más: de un Paleolítico Medio autóctono en nuestros grandes medios fluviales, sobre todo de recursos cuarcíticos, generado desde un pleno Achelense, Superior y Final transicional. Dados este proceso de génesis, las peculiaridades con que se nos presentan sus industrias y su extensión en las cuencas fluviales de la Península, creo que puede calificarse de Musteriense Ibérico. Desde él tendría explicación, por ejemplo, el llamado Vasconiense, como resultado del impacto de este Musteriense Ibérico en los complejos clásicos vascocantábricos, como un ejemplo regional de las interrelaciones entre ambos modos culturales del Paleolítico Medio de nuestra Península. 


\section{BIBLIOGRAFÍA}

Diaz del Olmo, F., Vallespi, E., 1988: "Secuencias fluvial y paleolítica del Guadalquivir: terrazas y conexiones arqueológicas (Sevilla)", Trabajos de Paleolítico y Cuaternario, Díaz DEL OLmo y VAlLeSPI (ed.), Sevilla, págs. 53-57.

Castiñeira, J., Garcian Rincón, M., Álvarez, G., Martin, J., 1988: “Estado actual de las investigaciones paleolíticas de la provincia de Huelva", Trabajos de Paleolítico y Cuaternario, DIAZ DEL OLMO y VALLESPI (ed.), Sevilla, págs. 7-25.

Giles, F., Santiago, A., 1988: "Avance al estudio del Paleolítico Inferior en la laguna de Medina, curso inferior del río Guadalete (Jérez de La Frontera, Cádiz)", Trabajos de Paleolítico y Cuaternario, DíAz DEL Olmo y VallesPí (ed.), Sevilla, págs. 131-149.

Querol, A., Santonja, M., 1973: “El yacimiento de cantos trabajados de El Aculadero (Puerto de Santa María)", Excavaciones Arqueológicas en España, n. ${ }^{\circ} 130$.

Rodríguez Vidal, J., Mayoral, E., Castiñeira, J., García Rincon, J. M., 1988: "Evolución geomorfológica del litoral suratlántico ibérico», Aluvionamientos cuaternarios de la depresión inferior del Guadalquivir, Aequa, Grupo Andaluz de Cuaternario, págs. 7-35.

SANTONJA, M. (e.p.): «El Paleolítico Inferior en España y Portugal». Journal of World Prehistory.

Vallespi, E., Álvarez, G., Díaz del Olmo, F., 1983-84: “Cantos talladios en la terraza baja del Guadalete, sector de Villamartín (Cádiz)", Boletín del Museo de Cádiz, IV, págs. 5-28.

VallesPi, E., 1986: “El Paleolítico Inferior y Medio en Andalucia». Homenaje a Luis Siret (1934-1984), Consejería de Cultura de la Junta de Andalucia, págs. 59-66. 
Vallespi, E., Diaz del Olmo, F., Álvarez, G., Vallespi Garcia, E., 1988: "Industrias y secuencia del Paleolítico Inferior y Medio en el Bajo Guadalquivir, provincia de Sevilla", Trabajos de Paleolítico y Cuaternario, Díaz DEL OLMO y VALLESPI (ed.), págs. 59-85. 